\title{
Pretilt EFFECT ON A VERTICALLY ALIGNED LIQUID CRYSTAL CELL FOR OPTICAL DISPLAYS
}

\author{
(Invited paper)
}

\author{
Volodymyr Tkachenko ${ }^{1}$, Giancarlo Abbate ${ }^{2}$, Antigone Marino ${ }^{3}$, Enrico Santamato ${ }^{3}$, \\ Noureddine Bennis ${ }^{4}$, Xabier Quintana ${ }^{4}$, Jose M. Otón ${ }^{4}$ \\ ${ }^{1}$ CNR-INFM Coherentia, clo Dip. Scienze Fisiche, \\ ViaCintia M. S. Angelo, Napoli, Italy,Fax:+39-081676342,e-mail: tkachenko@na.infn.it \\ ${ }^{2}$ CNR-INFM LiCryL and Coherentia, and Università di Napoli Federico II, \\ ViaCintia M. S. Angelo, Napoli, Italy, Fax:+39-081676342, ,e-mail: abbate@na.infn.it \\ ${ }^{3}$ CNISM and Università di Napoli Federico II, Via Cintia M. S. Angelo, \\ Napoli, Italy, Fax:+39-081676342, e-mail: antigone@na.infn.it \\ ${ }^{4}$ Universidad Politécnica de Madrid (UPM), Ciudad Universitaria, E-28040, \\ Madrid, Spain, Fax:+34-913367319, e-mail: n bennis@,tfo.upm.es
}

\begin{abstract}
A nonzero pretilt angle is generally used in vertical aligned (VA) mode liquid crystal (LC) displays to avoid domain formation during molecular reorientation by electric field, as well as to reduce both driving voltage and response time. The pretilt effect on electro-optical properties of a VA LC cell has been investigated. Pretilt was controlled changing the angle of $\mathrm{SiO}_{\mathrm{x}}$ deposition on the substrate surfaces. The tilt angle distribution versus the applied voltage is obtained by Variable Angle Spectroscopic Ellipsometry and compared with the simulated curves. A good compromise between contrast and response time is reached in cells with different pretilt values on the two cell substrates.
\end{abstract}

Keywords: liquid crystals, optical display, alignment layer, pretilt, ellipsometry

Vertical aligned (VA) mode liquid crystal (LC) cells are very popular in transmissive direct-view and reflective projection displays $[1,2]$ because of high contrast ratio and wide viewing angle. The contrast of pure VA cells is only limited by the quality of the polarizers. However, the LC orientation upon electric switching is undefined, therefore the cell usually generates domains after an extremely long delay. This situation can be avoided inducing a certain pretilt angle on the surface, so that the cell is given a preferred switching direction. The wider the angle from normal, the faster the switching. On the other hand, increasing the pretilt the quality of the dark state and consequently the contrast ratio will be reduced, and thus an optimal value of pretilt has to be sought.

Different pretilt angles were induced by changing the angle of $\mathrm{SiO}_{\mathrm{x}}$ thermal evaporation on the ITO coated glass substrates [3]. Average thickness of the $\mathrm{SiO}_{\mathrm{x}}$ layer was about $20 \mathrm{~nm}$. Coated substrates with antiparallel evaporation directions were assembled into sandwich cells with a gap of $4 \mu \mathrm{m}$ filled with MLC6608 (Merck) nematic LC mixture (see Fig. 1).

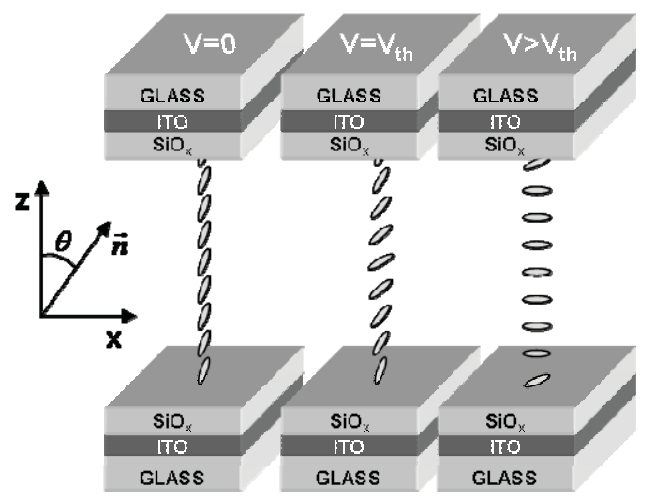

Figure 1- Scheme of the VA LC cell and molecule distributions inside for different voltages. 
Orientation of a nematic director $\vec{n}$ is characterized by the tilt angle $\theta$ from normal. At oblique deposition the $\mathrm{SiO}_{\mathrm{x}}$ layer has the sand dune like shape which is responsible for LC alignment with the pretilt angle $\theta_{0}$ on a substrate. Cells with $\mathrm{SiO}_{\mathrm{x}}$ deposition angles 50, 67, 70, and $80^{\circ}$ have been fabricated including the case of different angles on the two cell substrates, giving rise to a director distribution non symmetric with respect to the central plane of the cell.

If square form alternative voltage $V$, which exceeds the threshold value $V_{\text {th }}$, is applied the nonlinear $\theta$ profile along the cell gap gives rise, as shown in Fig. 1. The electro-optical response has been studied in transmissive mode by placing cells between crossed polarizers. Obtained values for contrast ratio (transmittance ratio in ON and OFF states), threshold voltage, and both rise and fall time (defined as the time interval between $10 \%$ and $90 \%$ values of the maximum transmittance) are presented in Table 1 .

Table 1- Electro-optical parameters for cells with different $\mathrm{SiO}_{\mathrm{x}}$ deposition layers

\begin{tabular}{|c|c|c|c|c|}
\hline $\begin{array}{c}\mathrm{SiO}_{\mathrm{x}} \text { deposition angles on } \\
\text { two substrates (degree) }\end{array}$ & $\begin{array}{c}\text { Contrast } \\
\text { ratio }\end{array}$ & $\begin{array}{c}\text { Threshold voltage } \\
(\mathrm{V})\end{array}$ & $\begin{array}{c}\text { Rise time } \\
(\mathrm{ms})\end{array}$ & $\begin{array}{c}\text { Fall time } \\
(\mathrm{ms})\end{array}$ \\
\hline $50-50$ & 110 & 2.75 & 5.0 & 5.0 \\
\hline $50-80$ & 80 & 1.75 & 3.6 & 5.2 \\
\hline $80-80$ & 10 & 1.00 & 0.5 & 6.8 \\
\hline
\end{tabular}

For the $\mathrm{SiO}_{\mathrm{x}}$ deposition angle of $80^{\circ}$ both response time and threshold voltage of a $\mathrm{LC}$ cell are reduced with respect to the case of $50^{\circ}$ but the contrast ratio become unacceptable. A good compromise has been found for the asymmetric cell "50-80": a good contrast ratio is maintained and less threshold and faster response are still provided.

To study the induced pretilt versus $\mathrm{SiO}_{x}$ evaporation angle optical characterization of cells has been performed by ellipsometry technique. The LC director profile across the cell and the pretilt angles were measured in two steps using the Variable Angle Spectroscopic Ellipsometer from J.A.Woollam Co. Inc. Measuring the complex reflection ellipsometry parameters $\Psi$ and $\Delta$ (Fig. 2a), we determined thickness of the cell and the average refractive indices of the liquid crystal [4]. The variation of the LC director inside the cell was obtained from the anisotropic transmission measurements varying the incident angle of the impinging light (Fig. 2b).

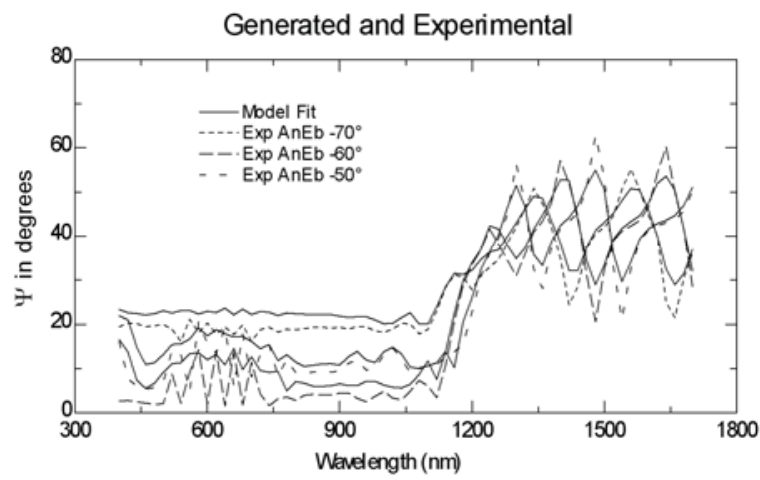

(a)

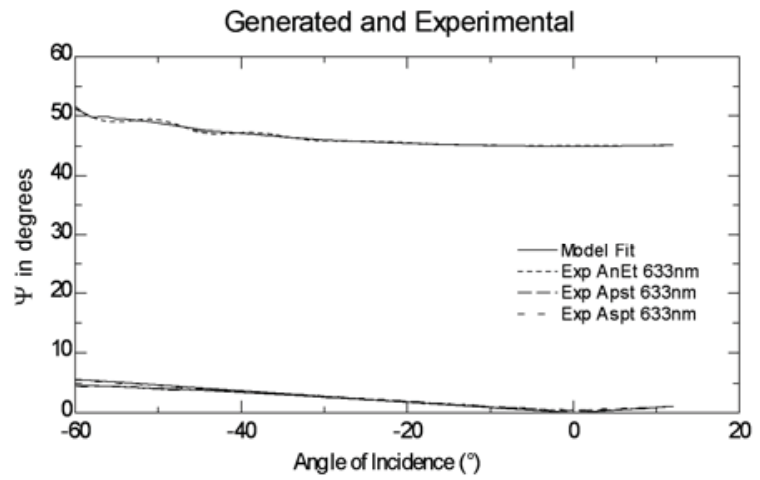

(b)

Figure 2- Ellipsometry angle $\Psi$ experimental (dashed lines) and generated (full lines) data for the "50-50" cell obtained in reflection (a) and transmission (b) acquisition modes.

Ellipsometry is very sensitive to LC director orientation but the results of measurements strongly depend on adequacy of a physical model [4]. Without electric field the tilt distribution has to be either uniform or linear. In this case the pretilt values have been 
measured with error of $1 \div 2^{\circ}$. A linear variation of the tilt angle occurs in asymmetric cells due to the different pretilt on two substrates, however, it is present in symmetric cells also, although less pronounced because of imperfect repeatability of the deposition process.

For the case of LC distorted by electric field the clear model for tilt distribution has to be found from theoretical considerations. By using a standard elastic theory, we have implemented a software program able to calculate the director profile inside the cell for varying input parameters, as pretilt angles, anchoring energy constants, driving voltage, including the possibility to provide different values at each surface to give account of asymmetric cells. For voltages below $3 \mathrm{~V}$ the profile has been found to be described by sinusoidal function.

Finally, the pretilt versus $\mathrm{SiO}_{\mathrm{x}}$ deposition angle obtained in this study is shown in Fig. 3 .

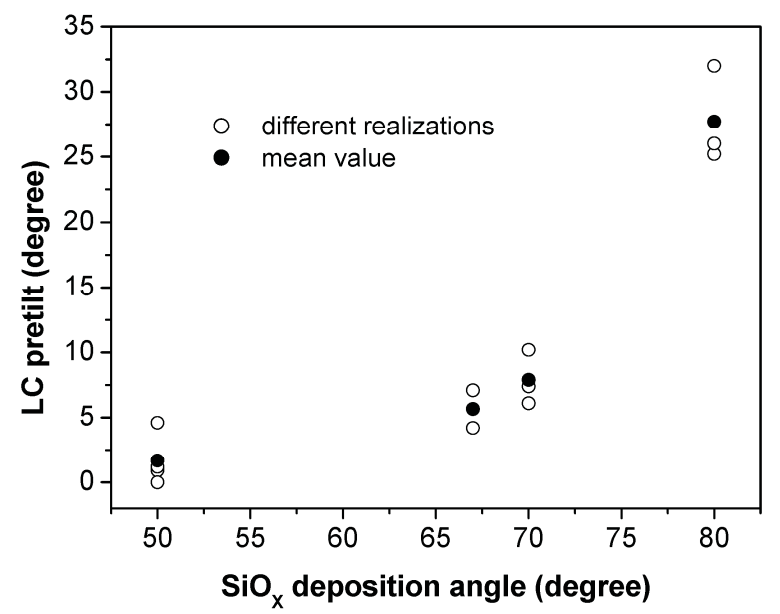

Figure 3- Pretilt versus $\mathrm{SiO}_{\mathrm{x}}$ deposition angle measured by ellipsometry

\section{REFERENCES}

1. J.J. Lyu, J. Sohn, H.Y. Kim, and S.H. Lee, J. Disp. Technol. vol. 3, p. 404, 2007.

2. D. Armitage, I. Underwood, and S.T. Wu, Introduction to Microdisplay. Wiley, New York, 2006.

3. X. Quintana, M.A. Geday, B. Cerrolaza, D. Pérez-Medialdea, J.M. Otón, Proc. 6TH IEEE Spanish Conf. on Electron. Dev. p. 277-280, 2007.

4. G. Abbate, V. Tkachenko, A. Marino, F. Vita, M. Giocondo, A. Mazzulla, L. De Stefano, Journal of Applied Physics vol. 101, p. 073105, 2007. 